
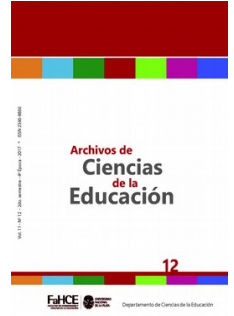

태들
Archivos de Ciencias de la Educación, Vol. 11, nº 12, diciembre 2017, e030. ISSN 2346-8866

Universidad Nacional de La Plata.

Facultad de Humanidades y Ciencias de la Educación.

Departamento de Ciencias de la Educación.

\title{
Nuevas tecnologías de la información como facilitadoras de aprendizaje significativo
}

\author{
New information technologies as facilitators of meaningful learning
}

\section{Irene Arriassecq* y Graciela Santos*}

* Universidad Nacional del Centro de la Provincia de Buenos Aires, Argentina | irenearr@exa.unicen.esu.ar; nsantos@exa.unicen.esu.ar

\section{PALABRAS CLAVE}

Aprendizaje

Enseñanza

Nuevas tecnologías

Didáctica

KEYWORDS

Learning

Teaching

New technologies

Didactics

\section{RESUMEN}

En este trabajo se propone abordar la potencialidad de las TIC para promover aprendizaje significativo asumiendo que, en la actualidad, existe consenso en la vigencia de ese marco teórico enriquecido con los diversos aportes teóricos que ha recibido desde la década del ' 60 y que, al mismo tiempo, las TIC posibilitan nuevas formas para el acceso a la educación dado que las instancias de aprendizaje se amplían ante una gran variedad de aplicaciones y recursos disponibles en Internet. Realizamos un análisis, desde el marco teórico del aprendizaje significativo, de tres aplicaciones informáticas para la enseñanza en las áreas de ciencias exactas y naturales: CMapTools, GeoGebra y Modellus en el contexto de una posible secuencia de enseñanza en el nivel secundario para el tópico de física "tiro parabólico".

\section{ABSTRACT}

This paper proposes to approach the potential of TIC to promote meaningful learning, assuming that, at present, there is consensus in the validity of this theoretical framework enriched with the various theoretical contributions it has received since the 1960s, At the same time, TIC enable new forms of access to education as learning institutions expand in the face of a wide variety of applications and resources available on the Internet. We performed an analysis, from the theoretical framework of significant learning, of three computer applications for teaching in the areas of exact and natural sciences: CMapTools, GeoGebra and Modellus in the context of a possible sequence of teaching at the secondary level for the topic of Physics "parabolic shot". 


\section{Introducción}

En 1963 Ausubel se refirió por primera vez al constructo aprendizaje significativo (AP) y, transcurrido más de medio siglo, no ha perdido vigencia: ha sido utilizado tanto por docentes como por diseñadores de currículos y ha servido de marco teórico de numerosas investigaciones en el área de Enseñanza de la Ciencia y de la Física en particular. A su vez, ha recibido aportes de diversos investigadores que han enriquecido el constructo, colaborando en su elucidación y aplicado en diversos contextos. Entre ellos podemos mencionar: la Teoría de la Educación de Novak (1988), la Teoría de la Educación de Gowin (1981) o el planteo de Moreira (2000 c), que integra la propuesta de Ausubel con la propuesta de enseñanza subversiva elaborada por Postman y Weingartner (1969). En diversas partes del mundo, investigadores de distintas áreas siguen sumando aportes que enriquecen este modelo. De hecho, se realizó en Argentina el VIII Encuentro Internacional sobre Aprendizaje Significativo, a veinticinco años del primer encuentro que se realizó en Cornell, EE.UU.

Los cambios en las prácticas humanas producto del constante avance de las TIC introducen cambios en los procesos sociales y en las pautas de actividad. En este sentido, la relación de las personas con la tecnología es bilateral (Burbules y Callister, 2008). Una concepción relacional de las TIC propone una mirada no solo sobre lo que se hace con la tecnología sino sobre las "pautas de uso" que se instalan en las prácticas sociales y producen un gran impacto social. Según Burbules y Callister, al hablar de las "nuevas” tecnologías debe quedar en claro que lo más nuevo tal vez no sea la tecnología sino todos los otros cambios que la acompañan.

Las TIC habilitan nuevas posibilidades para el acceso a la educación. Las instancias de aprendizaje se amplían ante las múltiples aplicaciones y recursos disponibles en Internet. Las aplicaciones específicas para la distribución y acceso a la información, la resolución de problemas y la comunicación aumentan las condiciones para configurar el aprendizaje significativo (Castro-Garcia et. al., 2016).

En educación sería un reduccionismo pensar que las herramientas TIC son promotoras de una transformación educativa si no cambian las prácticas educativas ni los enfoques didácticos, pedagógicos, psicológicos, etc. Por eso, en este artículo se ha querido llamar la atención sobre los cambios en las prácticas educativas que promueven las TIC y que son andamios para el aprendizaje significativo.

Teniendo en cuenta la vigencia y los aportes realizados desde el marco teórico del AS, nos surge el interrogante de si las TIC tienen el potencial para promover ese tipo de aprendizaje. Desde la perspectiva de este marco teórico realizaremos un análisis de aplicaciones educativas. En particular, tomando en consideración las aplicaciones informáticas para la enseñanza en las áreas de Ciencias Exactas y Naturales, disponibles en las netbooks que desde el Ministerio de Educación y Deportes de la Nación se han distribuido, y se distribuyen, en las escuelas públicas de nuestro país, abordaremos el estudio de: CMapTools, GeoGebra y Modellus.

\section{¿Cuáles son las “nuevas” tecnologías de la información utilizadas en educación?}

Las TIC son instrumentos óptimos para apoyar actividades de aprendizaje cuyo propósito es integrar distintos sistemas semióticos que amplían las posibilidades cognitivas (Coll y Martí, 2001). También son útiles para personalizar los aprendizajes de acuerdo a las necesidades específicas de cada alumno.

A la tecnología se le atribuye un rol transformador de la educación, como si estuviese en su naturaleza. Sin embargo, el fenómeno educativo involucra al estudiante, los conocimientos disciplinares y al docente. Las TIC tienen una enorme potencialidad para el desarrollo de innovaciones educativas dado que una de sus funciones primordiales es constituirse en instrumentos cognitivos para auxiliar en el proceso de aprendizaje. 
Pueden entenderse como una extensión de la mente humana que se ocupa de procesos rutinarios como el cálculo, ampliar la capacidad de memoria de largo plazo para almacenar información, o la memoria de corto plazo para trabajar con procesos que requieren manejar mucha información (Pea, 2001).

Con frecuencia la integración de las TIC en las prácticas educativas se impone a partir de un recurso o aplicación específica, por ejemplo, cuando se elabora una actividad de aprendizaje para utilizar determinada aplicación. Desde esta visión instrumentalista se subordina la práctica educativa al aprendizaje de una aplicación informática, perdiendo el sentido de uso de la tecnología.

El conocimiento requerido para enseñar con tecnología se obtendría de la sinergia entre el conocimiento del contenido, el conocimiento pedagógico y el conocimiento tecnológico (Harris y Hofer, 2009). La planificación debería seguir organizándose en torno a los requisitos de los diseños curriculares, las prácticas pedagógicas y, por último, en función de las posibilidades y limitaciones de las tecnologías disponibles. Con esto tampoco se quiere decir que el docente no deba enseñar conocimiento tecnológico, sino que su uso debe subordinarse a los contenidos curriculares a desarrollar.

La experiencia muestra que los estudiantes de física suelen tener dificultades para comprender conceptos abstractos basados solamente en representaciones proposicionales. Según Orozco Rodríguez, la comprensión de conceptos teóricos puede mejorar cuando se utilizan objetos de aprendizaje digitales (Orozco Rodríguez et. al., 2016).

Se denomina objeto de aprendizaje LO (Learning Objects) a un recurso multimedia que comprende una unidad pedagógica significativa que responde a un objetivo, una actividad de aprendizaje y una evaluación. Se puede reutilizar y combinar con otros LO cumpliendo ciertas especificaciones pedagógicas y tecnológicas. Poseen tres características claves que promueven la mediación de los aprendizajes significativos: el soporte visual que, al requerir menos memoria de trabajo y reducir la carga cognitiva, ayuda a la comprensión de ideas complejas y abstractas; la motivación, a través de un mayor enfoque; y el control del aprendizaje (Kay y Knaack 2008). Por ejemplo, un LO con baja interactividad y escaso control, utilizado para motivar o demostrar, tendrá menor impacto sobre el aprendizaje que uno empleado para enseñar un concepto nuevo. Por otro lado, también se considera que para llegar a esta instancia primero se debería analizar la potencialidad de la aplicación o recurso e identificar el aprendizaje que se podría profundizar.

No obstante, las tecnologías no son inocuas. Proponen otras formas de hacer las cosas e impactar en los modos de trabajar en el aula (Adell y Castañeda, 2012), tomando en cuenta la potencialidad para la personalización, el aprendizaje activo, el aprendizaje colaborativo y el aprendizaje autónomo o autodirigido. No siempre buenos LO garantizan que el alumno adquiera aprendizajes significativos (Rahmani, 2016), es necesaria la intervención adecuada y just in time del docente (Novak et al., 1999).

La interactividad es la característica distintiva de la tecnología que habilita cambios en las prácticas educativas, y que permite representar, visualizar, modelizar, probar hipótesis, procesar, buscar y gestionar información, compartir, comunicarse con otros, etc. El proceso de interacción que se genera es sumamente complejo y requiere que se establezca el diálogo y adaptabilidad entre el alumno y la aplicación (Miranda, 2014).

La integración de las TIC a las prácticas educativas permite vincular los contenidos, las estrategias de enseñanza y los procesos de aprendizaje. Por ejemplo, incorporar un objeto de aprendizaje elaborado con la aplicación GeoGebra que facilite la comprensión (Orozco Rodríguez, 2014; Malgieri, 2014), utilizar una simulación (Ariza y Quesada, 2014) o un mapa conceptual para elaborar una síntesis (Novak y Cañas, 2006), etc. En particular, las aplicaciones informáticas para elaborar mapas conceptuales son concebidas como herramientas didácticas y metacognitivas que pueden facilitar la negociación de significados y, al mismo 
tiempo, reflejar la forma en que un alumno ha conceptualizado un determinado contenido en un cierto momento.

Se intenta presentar una perspectiva de las aplicaciones informáticas como mediadora de aprendizajes significativos, superadora de la noción de recurso didáctico. Para esto se han tomado en consideración las ideas socioculturales de la cognición humana, el rol mediador de las herramientas en las acciones que desenvuelven las personas sobre el mundo y la relevancia de la actividad que las motiva.

Sería deseable que la intencionalidad pedagógica privilegie la comunicación, la autonomía en el aprendizaje, la obtención, selección y análisis crítico de la información y la resolución de problemas en contexto. Y, además, que el sentido de uso esté asociado a la potencialidad mediadora de las TIC para modificar procesos intra e inter-psicológicos implicados en la enseñanza y el aprendizaje (Coll, 2011).

\section{Análisis de las nuevas tecnologías a partir de la Teoría del Aprendizaje significativo}

El aprendizaje significativo es un proceso a través del cual se logra relacionar un nuevo conocimiento, o nueva información, mediante la interacción con la estructura cognitiva del sujeto que aprende; en particular, con las denominadas ideas de anclaje o subsumidores que son ideas, conceptos o proposiciones claras e inclusivas, disponibles en la mente de quien intenta aprender y que permiten otorgar significado al nuevo contenido cuando interactúa con las ideas que el sujeto posee. En el proceso de interacción, también se modifican los subsumidores que resultan más diferenciados, elaborados y estables (Ausubel, 2002; Moreira, 2000 a).

Para que se logre un aprendizaje significativo es necesario que se cumplan ciertas condiciones referidas tanto al sujeto que aprende como al material de trabajo que se utilice.

En cuanto a la persona que aprende, para lograr específicamente un aprendizaje significativo, es necesario que manifieste predisposición para aprender de forma significativa. Esto significa que quien se encuentra en situación de aprender debe intentar establecer relaciones sustantivas y no arbitrarias entre su estructura cognitiva y el material de estudio. Como consecuencia, es necesario que el individuo cuente en dicha estructura con los subsumidores adecuados.

Respecto del material utilizado, debe ser potencialmente significativo, es decir debe tener un significado lógico potencialmente relacionable, de forma no arbitraria y sustantiva con la estructura cognitiva del sujeto (Ausubel et al., 1991, Moreira, 2000 b). Cuando esas condiciones no se cumplen, el tipo de aprendizaje logrado es mecánico, no significativo.

Es importante entonces realizar un análisis conceptual de los contenidos que se aborden y de las relaciones existentes entre los mismos. También es fundamental organizarlos secuencialmente y, considerando que el alumno no aprende un contenido con sólo tomar contacto una vez con el mismo, es necesario que los materiales que se le proporcionen contemplen sucesivas aproximaciones a los contenidos, reiteraciones y revisiones permanentes. Bajo estas condiciones, el alumno puede ir discriminando significados de los conceptos involucrados, corregir y negociar significados en la interacción permanente con el docente, sus compañeros y el material.

Para Ausubel, los conceptos constituyen un aspecto central de su teoría y los define como: “... objetos, acontecimientos, situaciones o propiedades que poseen atributos de criterio comunes y que están diseñados en cualquier cultura dada mediante algún signo o símbolo aceptado” (Ausubel et al., 1991, p. 88).

La teoría de Ausubel se centra principalmente en el aprendizaje que se produce en un contexto educativo, fundamentalmente de los conceptos científicos a partir de los conceptos que el niño previamente ha formado 
en su vida cotidiana. Asume como un rasgo fundamental de su teoría que el conocimiento se organiza en estructuras y que se producen reestructuraciones como resultado de la interacción entre esas estructuras, presentes en el sujeto, y la nueva información. Es justamente en ese aspecto donde la instrucción formal desempeña un rol fundamental como desestabilizante de las estructuras existentes. Moreira (2000 a) señala que las personas científicamente educadas comparten significados científicamente aceptados y en forma consciente pueden discriminar entre esos significados y aquellos que no son aceptados en el ámbito científico. Y respecto de los sujetos que se inician en cualquier campo de conocimiento manifiesta:

Cuando ellos aprenden por primera vez los significados contextualmente aceptados sus significados alternativos todavía son más estables y dominantes. A medida que ocurre el aprendizaje significativo, la concepción se desarrolla y aumenta la discriminabilidad; no obstante, significados ya establecidos no son reemplazados o borrados: ellos pueden quedar cada vez menos utilizados, o no utilizados, pero todavía siguen presentes en la concepción que se desenvuelve (y queda más rica), tal vez "escondidos" en algunos significados residuales (p.310).

Ausubel discrimina entre dos formas básicas para adquirir conceptos, esto es, de relacionar determinados objetos o eventos con ciertos atributos comunes a todos ellos. Una forma se da mediante la formación de conceptos que consiste en abstraer inductivamente a partir de experiencias empíricas concretas (no verbales). Incluye en este tipo de aprendizaje procesos como la diferenciación, la generalización, la formulación de hipótesis, etc. Se trata de la forma característica en que los niños en edad pre-escolar, incluso durante los primeros años de la escolarización, adquieren conceptos y proposiciones. La otra es la asimilación de conceptos, que consiste en relacionar nuevos conceptos con otros anteriormente formados y disponibles en la mente del niño. Se produce por recepción (no por descubrimiento) en contextos de instrucción formal. Este tipo de aprendizaje sería el principal durante la edad escolar, particularmente en la adolescencia y en la edad adulta. Es un aprendizaje significativo ya que:

... puede incorporarse a las estructuras de conocimiento que posee el sujeto a partir de su relación con conocimientos anteriores. Para ello es necesario que el material que debe aprenderse posea un significado en sí mismo, es decir, que haya una relación no arbitraria... entre sus partes... es necesario además que el alumno disponga de los requisitos cognitivos necesarios para asimilar ese significado (Ausubel, 2002., p. 37).

A su vez, Ausubel distingue tres formas de aprendizaje por asimilación:

- Aprendizaje subordinado: puede consistir en una inclusión derivativa donde la nueva información se vincula, y representa, a un caso particular de una idea supraordenada; o en una inclusión correlativa, donde el nuevo concepto se relaciona con otro existente, aunque puede ser considerado una extensión, modificación o limitación del mismo. En este tipo de aprendizaje se produce una diferenciación progresiva de los conceptos ya existentes y es, para Ausubel, la principal forma de adquirir conocimiento (Pozo, 1989).

- Aprendizaje superordenado: en este tipo de aprendizaje, ciertos conceptos ya establecidos son considerados como ejemplos específicos de uno nuevo con el que se relacionan. Este nuevo concepto se define con un conjunto de atributos de criterio que abarcan las ideas subordinadas. Es un proceso inverso a la diferenciación. Se produce una reconciliación integradora entre los rasgos de una serie de conceptos dando lugar a la formación de un concepto más general o supraordenado. 
- Aprendizaje combinatorio: en este caso, el nuevo concepto se relaciona con los ya existentes aunque no de forma jerárquica (no es más inclusivo ni específico que éstos). Sin embargo, se asume que el nuevo concepto comparte algunos atributos de criterio con los preexistentes.

Como señalábamos anteriormente, desde que surgió el constructo aprendizaje significativo, muchos investigadores realizaron aportes al mismo, enriqueciéndolo. Moreira (2000 c), por ejemplo, plantea la idea de promover en la escuela un aprendizaje significativo crítico integrando presupuestos de Ausubel, Postman y Weingartner (1969 en Moreira ibíd.). Desde esta perspectiva, aprender implica una actitud crítica del sujeto hacia su propio proceso de aprendizaje y respecto del contenido que se aborda. Quien intenta aprender significativa y críticamente un contenido debería cuestionarse qué es lo que desea aprender, por qué debería aprenderlo y para qué. Un alumno capaz de lograr un aprendizaje con estas características tendrá los elementos necesarios para posicionarse dentro del contexto sociocultural al que pertenece de forma crítica y analítica, permitiéndole tomar decisiones e implementarlas; aprenderá a manejar el conocimiento de la cultura en la que se encuentra inmerso sin que éste lo domine. Habrá logrado un aprendizaje crítico y subversivo:

Parafraseando a Postman y Weingartner (1996), mi argumento es que ese foco debería estar en el aprendizaje significativo subversivo, aprendizaje que permitirá al sujeto formar parte de su cultura y, al mismo tiempo, estar fuera de ella, manejar la información críticamente, sin sentirse impotente; usufructuar la tecnología sin idolatrarla; cambiar sin ser dominado por el cambio; convivir con la incertidumbre, la relatividad, la causalidad múltiple, la construcción metafórica del conocimiento, la probabilidad de las cosas, la no dicotomización de las diferencias, la recursividad de las representaciones mentales; rechazar las verdades fijas, las certezas, las definiciones absolutas, las entidades aisladas (Moreira, 2000 c, p. 13).

Otro aspecto interesante de este planteo, es que el error es aceptado como natural y debe ser superado en el proceso de aprendizaje. Cuando un alumno deber enfrentarse con un nuevo conocimiento para el cual los subsumidores que posee son inadecuados, pero aprendidos significativamente, debería poder recurrir a su sentido crítico para no utilizarlos (p. 23). Según Moreira, es necesario que se realice un desaprendizaje del conocimiento previo.

La teoría de Ausubel (2002) proporciona, además, principios programáticos facilitadores (la diferenciación progresiva, la reconciliación integradora, la organización secuencial y la consolidación) y algunas estrategias facilitadoras (los organizadores previos, los mapas conceptuales y los diagramas de " $V$ " -Novak y Gowin, 1999; Moreira y Buchweitz, 1993-). Para la elaboración de nuestra propuesta didáctica tomamos en cuenta algunos aspectos del modelo que propone Ausubel para el aprendizaje significativo y del planteo realizado por Moreira para un aprendizaje crítico, que se integran al marco didáctico adoptado.

Consideramos, al igual que Ausubel (2002), que los libros de texto utilizados habitualmente -por docentes y alumnos- en la escuela no poseen una organización jerárquica global que contemple las relaciones y conexiones entre diferentes temas. Por otra parte, se evita en los mismos la reiteración, entendiéndola como un aspecto negativo.

Según Ausubel, el docente es quien crea y/u organiza los materiales potencialmente significativos que se implementan en el aula. En un aprendizaje significativo, se está asumiendo que intervienen un sujeto que aprende y otras personas que interactúan con él, fundamentalmente el docente y otros alumnos.

En el aprendizaje de conceptos, tanto por formación como por asimilación, el rol del docente es fundamental. En el primer caso, el docente informa al alumno cuál es la palabra o signo que corresponde al objeto o 
evento que se está analizando. En el caso de la asimilación de conceptos, el alumno la realiza a partir de conceptos que ya existen en su estructura cognitiva en una interacción con el docente mediador.

Si bien ya hemos mencionado autores que han enriquecido el constructo aprendizaje significativo debemos agregar que, además, han resignificado el rol del docente.

Novak (1988, 1998) plantea la necesidad de negociar significados entre alumnos y docentes durante el proceso educativo en el que se pretende un aprendizaje significativo.

Gowin (1981) destaca la importancia de comprobar el significado que los alumnos han construido en el contexto del aula, mediante la interacción personal entre el docente y los alumnos con el objetivo de compartir significados.

Moreira (2000 c) plantea que un aprendizaje significativo crítico se propicia a través de preguntas relevantes y que las respuestas surgen en el intercambio y negociación de significados que los alumnos realizan entre sí y, fundamentalmente, con el docente.

En nuestra propuesta rescatamos las siguientes categorías -vinculadas tanto a la propuesta original de Ausubel como a las diversas contribuciones a la misma- que guían el análisis de la potencialidad de las TICs para promover aprendizaje significativo:

- Promoción de interacciones: el aprendizaje significativo que pueden lograr los alumnos en situación de clase está condicionado fuertemente por el tipo de interacciones que se logren propiciar entre los alumnos y el docente y, además, entre los propios alumnos. Se analiza la posibilidad de que los objetos de aprendizaje permitan la interacción del alumno con el contenido, del alumno con sus pares y del alumno con el docente.

- Potencial motivacional: es indispensable que el alumno esté dispuesto a aprender para lograr un aprendizaje significativo. La interacción del estudiante con los componentes de la interfaz, que en muchos casos muestran una representación dinámica del funcionamiento de un sistema o la visualización de procesos, brindan respuesta inmediata del sistema a sus acciones (feedback inmediato) que le permiten constatar o verificar sus inferencias. Por este motivo, se analiza el aspecto visual, dado que es posible incluir tanto imágenes estáticas como dinámicas en los objetos de aprendizaje.

- Capacidad para elucidar ideas previas: se analiza si el software permite explicitar ideas previas, ya que son necesarias para analizar si el alumno cuenta con los subsumidores adecuados.

- Organización del contenido: se analiza si el objeto de aprendizaje es conceptualmente claro y los lenguajes de representación que utiliza pueden relacionarse con el conocimiento previo del alumno.

A continuación, se presenta una breve descripción de las tres aplicaciones informáticas seleccionadas para evaluar su potencial en pos de promover el aprendizaje significativo. Los tres softwares seleccionados son de distribución gratuita y se pueden descargar a través de Internet.

El software CmapTools $\underline{\underline{1}}$ se diseñó con el objeto de apoyar la construcción de modelos de conocimiento representados en forma de Mapas Conceptuales (Cañas et al., 2004; Novak y Cañas, 2006). Consta de un conjunto de herramientas que ayudan a construir mapas conceptuales manipulando objetos en pantalla a partir de operaciones simples de arrastrar y soltar. Además, permite vincular distintos recursos (imágenes, videos, texto, páginas web, documentos, presentaciones, etc.) a los mapas conceptuales; trabajar de manera 
conjunta con los conceptos, las palabras de enlace o las conexiones; armar proposiciones; visualizar el esquema del mapa y grabar su construcción. El software también facilita el intercambio de los mapas conceptuales elaborados. Estas funcionalidades exigen que el estudiante realice nuevas acciones cognitivas. Asimismo, abre la posibilidad de repensar los mapas conceptuales y de comprometerse en la construcción de las estructuras de conocimiento que lo conduzcan a un aprendizaje significativo.

Modellus $^{2}$ es un software que posibilita la producción de representaciones alternativas de un modelo en forma de simulación analógica con animaciones, visualizando las relaciones mediante gráficos y tablas. Esta funcionalidad ayuda al estudiante a analizar (pensar) el fenómeno sin necesidad de manipular (en los primeros momentos de interacción con el software) ecuaciones complejas a las que, habitualmente, les resulta difícil asignarles un significado. Una de las ventajas de este software es que el estudiante, a medida que modela, puede ir constatando la validez de la representación con la herramienta animación e ir corrigiendo su modelo mental; lo que le brinda cierta autonomía para realizar elaboraciones significativas.

El tercer software seleccionado es GeoGebra ${ }^{3}$ de matemática dinámica que permite trabajar con geometría, álgebra y cálculo. Las ecuaciones y coordenadas se pueden introducir directamente desde el teclado. Trabaja con variables vinculadas a números, vectores y puntos para calcular derivadas e integrales de funciones. Proporciona un conjunto de comandos de análisis específicos de matemáticas para identificar puntos individuales de una función, tales como raíces y extremos. Se puede visualizar el objeto matemático en tres representaciones diferentes: gráfica (como en el caso de puntos y gráficos de funciones), algebraica (como coordenadas de puntos, ecuaciones) y hoja de cálculo. Cada representación del objeto puede unirse a la otra en una adaptación dinámica entre sí, absorbiendo los cambios producidos en cualquiera de ellos.

\section{Análisis de las aplicaciones a partir de una situación didáctica}

Un juego muy conocido (y consumido) tanto por niños como por adultos, es el Angry Birds. La meta de cada nivel del juego es deshacerse de unos cerdos. Los cerdos usualmente están rodeados por madera, cristal, piedra u otros materiales que están organizados en estructuras. El jugador debe arrojar los pájaros enojados usando una honda e intentar que impacten en la estructura que contiene a los cerdos para deshacerse de ellos.
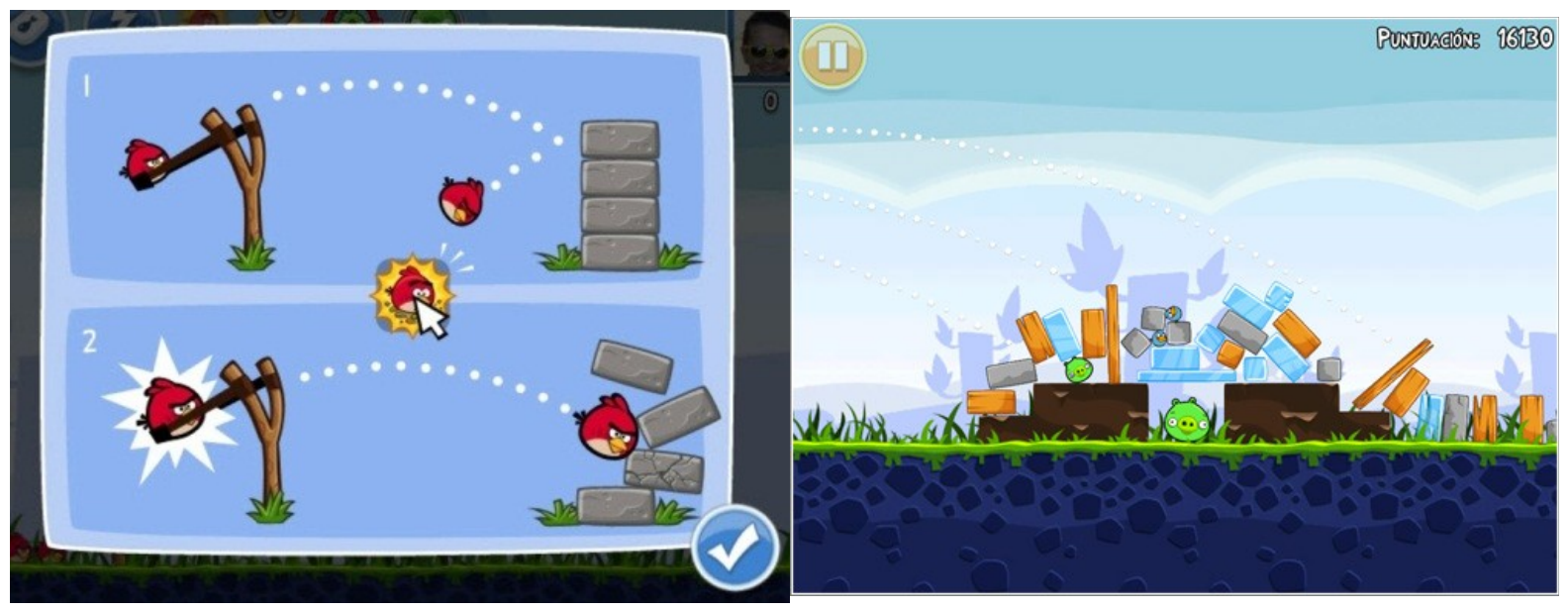

Angry Birds es una serie de videojuegos creada en 2009 que se adapta a sistemas operativos de PC y a dispositivos de pantalla táctil, como los basados en Maemo, iOS, Symbian, Java y Android. Tuvo más de mil millones de descargas en 2012, fue la aplicación más popular del año y el juego más vendido de la historia en soportes móviles. Los personajes son tan populares que la empresa creadora de la aplicación incluso comercializa juguetes de los pájaros, disfraces y todo tipo de mercancía relacionado con el juego. En el 2016 
se lanzó una adaptación cinematográfica homónima, basada en la franquicia de juegos Angry Birds, que contó con la participación de actores famosos para dar vida a los personajes.

Este juego, que es altamente probable que conozcan y jueguen los estudiantes, podría constituirse dentro del aula en un recurso que promueva la motivación necesaria para abordar significativamente conceptos de física. En nuestro caso, lo utilizaremos para abordar el tópico tiro oblicuo o movimiento parabólico.

Generalmente, el tema tiro oblicuo o movimiento parabólico se analiza en las clases de física de la escuela secundaria en la unidad sobre cinemática, o bien, en física básica a nivel universitario. Sin embargo, consideramos que sería pertinente abordarlo cuando los estudiantes hayan construido conocimiento tanto de cinemática como de dinámica, de modo de comprender por qué el análisis del tiro parabólico requiere la descomposición en dos tipos de movimientos distintos.

En la secuencia didáctica que analizamos se asume que los estudiantes conocen el juego Angry Birds como así también las herramientas informáticas antes descriptas. De no ser así, se permite que se familiaricen tanto con el juego como con las aplicaciones. Luego, se propone utilizar el CMapTools para construir un mapa conceptual como primera tarea. Se proporciona a los estudiantes los siguientes conceptos que deben incluir en dicho mapa: tiro parabólico, trayectoria, velocidad, aceleración, fuerza, peso, alcance, altura máxima y sistema de referencia. La pregunta de enfoque es: ¿Cómo puede ayudarnos la física a ganar más puntos en el Angry Birds? La herramienta utilizada para construir el mapa conceptual solicitado permite que el alumno interactúe con el contenido, con otros compañeros -dado que es posible trabajar en línea-, con el docente y con el resto de la clase al compartirlo e intercambiar significados. En cuanto al aspecto motivacional, la herramienta permite el trabajo creativo, incorporar imágenes y videos, incluso de los propios logros del alumno con el juego.

A partir de los conceptos seleccionados por el docente y la pregunta de enfoque, es posible elucidar las ideas previas del estudiante respecto de los conceptos de cinemática y mecánica que son necesarios para comprender significativamente el tiro oblicuo. Al mismo tiempo, es posible analizar de qué forma el alumno organiza los contenidos de cinemática y dinámica y si esa organización puede permitirle abordar un problema nuevo.

Una vez que han explicitado, discutido y acordado conocimientos acerca de las relaciones entre los conceptos de cinemática y dinámica (posición, velocidad, aceleración y fuerza), se les propone profundizar en dichas relaciones utilizando los programas GeoGebra y Modellus. Para trabajar en estos programas con animaciones dependientes del tiempo, debe destacarse una diferencia importante entre ambos: Modellus posee una ventana de animación, que representa al plano $x y$, donde es posible insertar objetos y asignarles comportamientos de movimiento que dependen del tiempo como variable independiente. Mientras que en GeoGebra no se cuenta con esta posibilidad. Por lo tanto, para representar al tiempo, es necesario crear un número libre y animar. Luego, al asociarle a los objetos los puntos de posición en función del tiempo, se logra simular el movimiento modelado. Tomando en cuenta las particularidades mencionadas de cada software y, en este caso, con el objetivo de utilizarlas para aumentar la comprensión del movimiento parabólico, se proponen tres instancias para estudiar dicho movimiento. En una primera instancia, en GeoGebra, se asigna a un punto A un movimiento rectilíneo uniforme sobre el eje $x$ y, a otro punto B, se le asigna un movimiento de tiro vertical sobre el eje $y$. Posteriormente, se crea un tercer punto $C$ y se le asigna a la coordenada $x$ el comportamiento de la abscisa del punto A y a la coordenada $y$ el comportamiento de la ordenada de B. De esta manera, los estudiantes están trabajando una composición de movimientos. 


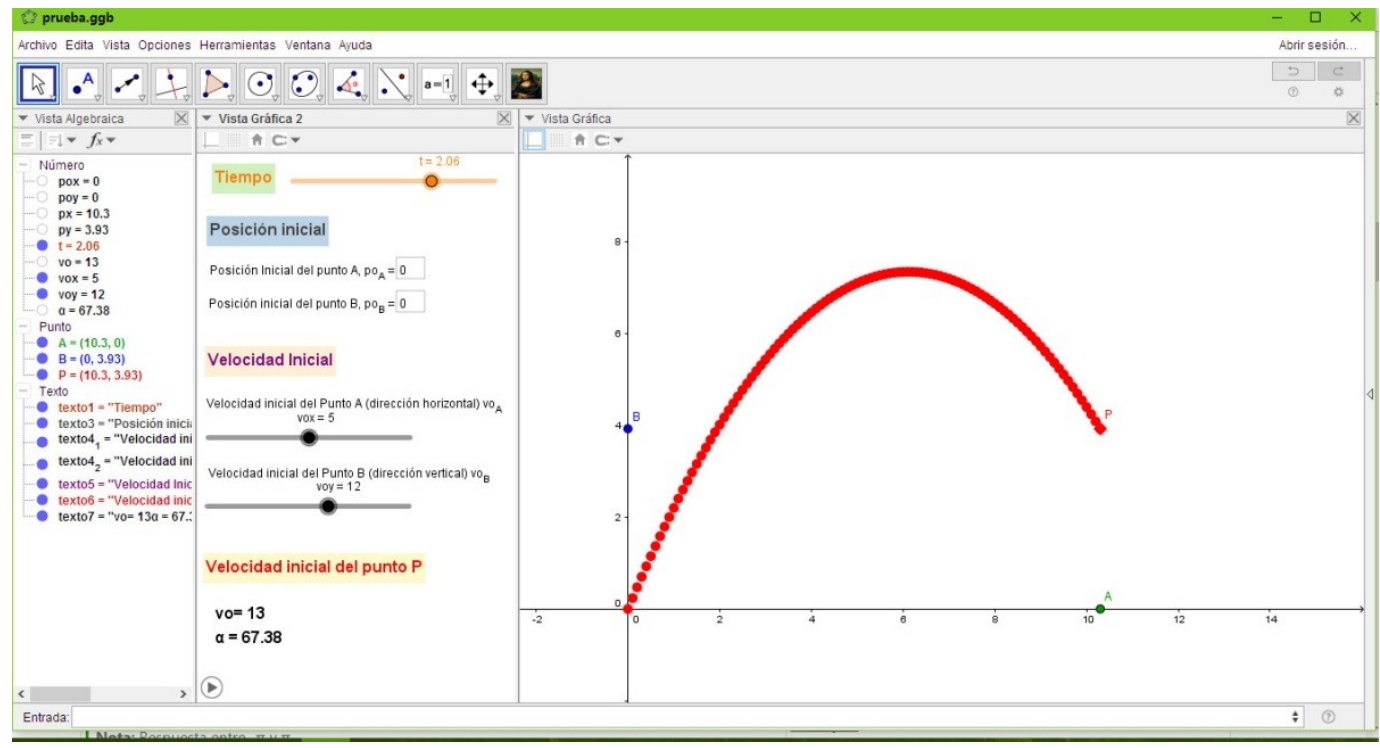

Figura 1. Pantalla en la que se muestra la construcción realizada en GeoGebra para componer el movimiento parabólico.

Para realizar la representación del movimiento resultante en Modellus, se requiere ingresar las ecuaciones de movimiento y así lograr una visualización del movimiento parabólico asignando ese comportamiento a un objeto y dando valores a los parámetros del modelo.

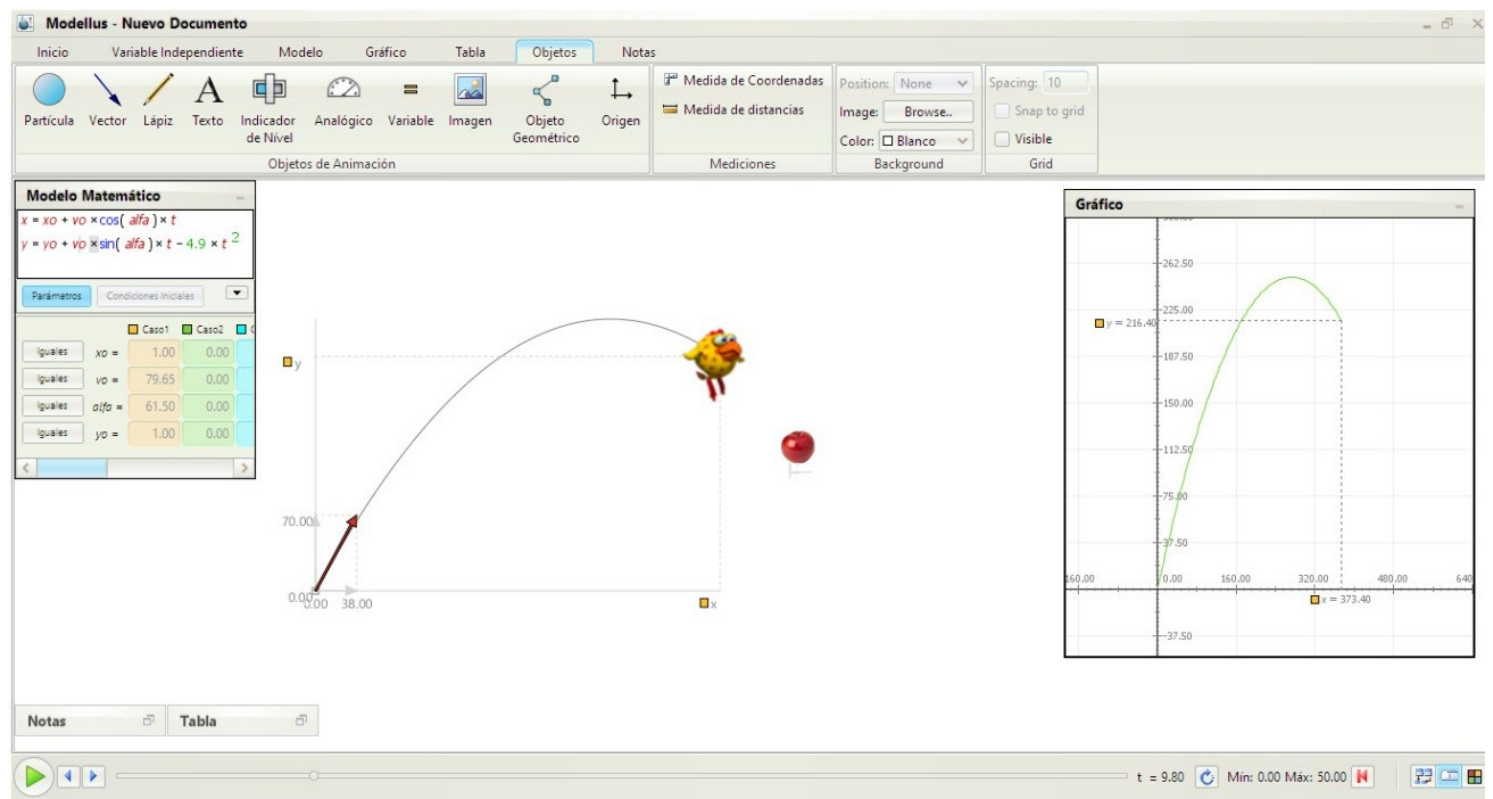

Figura 2. Pantalla con el modelado del videojuego Angry Birds con el software Modellus.

En síntesis, con el software GeoGebra se trata de relacionar dos movimientos conocidos y componer el movimiento parabólico del punto P. En esta construcción se trabaja en coordenadas cartesianas y se calculan las coordenadas polares de la velocidad inicial del movimiento parabólico (vo y $\alpha$ ). Este concepto se utiliza para modelar el movimiento parabólico con el software Modellus en coordenadas polares. Esta estrategia ayuda al estudiante a comprender la composición de movimientos, pero también a comprender los efectos de 
variar el ángulo e intensidad de lanzamiento (variando el vector, ver Figura 2) en el movimiento parabólico para que se cumpla el objetivo: "ganar puntos alcanzando a un objeto con otro que se lanza".

Con esta actividad se pretende ejemplificar la potencialidad de las herramientas informáticas para promover AS. En relación a las categorías mencionadas previamente, se puede destacar que Modellus y GeoGebra promueven interacciones con el contenido que pueden ayudar a diferenciar los movimientos, comprender las particularidades de los mismos e integrarlos en un nuevo conocimiento. Además, se favorecen los debates e interacciones con sus pares y con el docente.

Para el estudiante es un elemento motivador el desafío de modelar un videojuego "familiar" o conocido y comprender las nociones físicas incluidas. Es interesante destacar que la tarea de modelar con estas herramientas informáticas implica una interpelación permanente de las ideas previas con las nuevas frente a la visualización que se recibe como feedback de las acciones que se realizan en pantalla.

Además, el planteo de la propuesta intenta obtener beneficio de los lenguajes de representación que utiliza cada programa. Es importante destacar que, aunque un software sea diseñado en el marco de la teoría de AS, será la actividad de aprendizaje propuesta el componente determinante para el AS.

\section{Comentarios finales}

Actualmente existe consenso en la vigencia del marco teórico del AP enriquecido con los diversos aportes que ha recibido desde la década del '60 hasta la actualidad. Al mismo tiempo, las TIC posibilitan nuevas formas para el acceso a la educación. De lo que resulta que las instancias de aprendizaje se amplían ante una gran variedad de aplicaciones y recursos disponibles en Internet.

Comenzamos este trabajo planteándonos como interrogante si las TIC tienen el potencial para promover AP y, para aproximar una respuesta, realizamos un análisis desde el marco teórico AP de tres aplicaciones informáticas para la enseñanza en las áreas de ciencias exactas y naturales: CMapTools, GeoGebra y Modellus y planteamos cómo incorporarlas para abordar un problema clásico de física para el nivel secundario.

El AS puede producirse en diversos contextos y con independencia del uso de TIC. Sin embargo, prescindir de este tipo de recurso, cuando en la actualidad están disponibles tanto para estudiantes como para docentes -en las aulas y fuera de ellas- sería desaprovechar una herramienta valiosa para potenciarlo. Las herramientas informáticas posibilitan que los estudiantes desarrollen un pensamiento crítico acerca del contenido objeto de estudio y habilitan diversas formas de abordar y representar los conceptos.

En la selección de LO es importante considerar el grado de interactividad, el modelo representado, el diseño de la interfaz y el grado de involucramiento que el recurso le exige al usuario. Sin embargo, sería necesario realizar estudios para conocer cómo estos componentes interactúan con el aprendizaje para que adquiera significatividad.

Sin duda, el docente debe estar formado no solo en un marco teórico como es el AS sino también en el uso de las TIC de manera que pueda incorporarlas, desde una perspectiva significativa y no arbitraria, en el diseño de secuencias didácticas.

\section{Notas}

1 Institute for Human and Machine Cognition (IHMC), de la Universidad de West Florida (Estados Unidos) http://cmap.ihmc.us/cmaptools/ 
2 http://modellus.co/index.php?lang=es

3 https://www.geogebra.org/

\section{Bibliografía}

Adell, J. y Castañeda, L. (2012). Tecnologías emergentes, ¿pedagogías emergentes? En J. Hernández, M. Pennesi, D. Sobrino y A. Vázquez (coord.). Tendencias emergentes en educación con TIC. Barcelona: Asociación Espiral, Educación y Tecnología.

Ariza, M .R. y Quesada, A. (2014). Nuevas tecnologías y aprendizaje significativo de las ciencias. Enseñanza de las Ciencias, 32 (1), 101-115.

Ausubel, D., Novak, J. y Hanesian, H. (1991). Psicología Educativa, un punto de vista cognoscitivo. México: Ed. Trillas.

Ausubel, D. (2002). Adquisición y retención del conocimiento. Una perspectiva cognitiva. Barcelona: Paidós.

Burbules, N y Callister, T. (2008).Educación: Riesgos y promesas de las nuevas tecnologías de la Información.BuenosAires: Editorial Granica.

Cañas, A., Hill, G., Carff, R., Suri, N., Lott, J., Eskridge, T. (2004). CmapTools: A Knowledge Modeling and Sharing Environment. In A. J. Cañas, J. D. Novak \& F. M. González (Eds.), Concept Maps: Theory, Methodology, Technology. Proceedings of the First International Conference on Concept Mapping (Vol. I, pp. 125-133). Pamplona, Spain: Universidad Pública de Navarra.

Castro-Garcia, D., Olarte Dussán, F. andCorredor, J. (2016).Technology for Communication and Problem Solving in the Classroom. Effects on Meaningful Learning. Digital Education Review, (30), 207-219.

Coll, C. (2011). Aprender y enseñar con las TIC: expectativas, realidad y potencialidades, en: Carneiro, R.; Toscano, J.C.; Díaz, T. Coord. (2011). Los desafíos de las TIC para el cambio educativo. Colección Metas Educativas 2021. OEI y Fundación Santillana.

Coll, C. y Martí, E. (2001). La educación escolar ante las nuevas tecnologías de la información y de la comunicación. En C. Coll, J. Palacios y A. Marchesi (Comp.), Desarrollo Psicológico y Educación 2. Psicología de la Educación Escolar. Madrid: Alianza.

Dede, C. (1998). Learning with technology. USA: Association for Supervision and Curriculum Development. Trad. español: DEDE C. 2000. Aprendiendo con tecnologia. Buenos Aires: Editorial Paidós.

Gowin, D. (1981). Educating. Ithaca, N. Y.: CornellUniversity Press.

Harris, J., \& Hofer, M. (2009). “Grounded” technology integration: Planning with curriculum-based learning activity types. Learning \& Leading With Technology, 37(2), 22-25.

Kay,R. and Knaack, L. (2008). A multi-component model for assessing learning objects. Australasian Journal of Educational Technology, 24(5), 574-591.

Malgieri, M., Onorato, P. and De Ambrosis, A, (2014). Teaching quantum physics by the sum over paths approach and GeoGebra simulations, Eur. J. Phys, 35 (5), 1-21.DOI: 10.1088/0143-0807/35/5/055024.

Miranda, A. (2014). La interacción digital en el aula. Los procesos de significación en el uso de simulaciones computacionales en la escuela secundaria. Tesis doctoral, Doctorado en Ciencias de la Educación de la Facultad de Filosofía y Humanidades de la Universidad Nacional de Córdoba. 
Moreira, M. A. (2000 a) Reporte final de la VII Conferencia Interamericana sobre Educación en Física. Canela, Porto Alegre, Brasil.

Moreira, M. A. (2000 b) Aprendizaje Significativo: teoría y práctica. Madrid: Visor.

Moreira, M. A. (2000 c) Aprendizaje Significativo Subversivo. Conferencia dictada en el III Encuentro Internacional sobre Aprendizaje Significativo, Lisboa (Peniche), 11 a 15 de septiembre de 2000.

Moreira, M. A. y Buchweitz, (1993). Novas estratégias de ensino e aprendizagem: os mapas conceptuais e o vê epistemológico. Lisboa: Plátano Edições Técnicas.

Novak, J. D. y Cañas, A. J. (2006). La Teoría Subyacente a los Mapas Conceptuales y Cómo Construirlos, Reporte Técnico IHMC CmapTools 2006-01, Florida Institute for Human and Machine Cognition, 2006. Recuperado el 25/05/15: http://cmap.ihmc.us/Publications/ResearchPapers/TheoryUnderlyingConceptMaps.pdf

Novak, G.M., Patterson, E. T., Gavrin, A. D., Christian, W. and Forinash, K. (1999). Just in Time Teaching. American Journal of Physics (67), 937. http://dx.doi.org/10.1119/1.19159.

Novak, J. (1988). Teoría y práctica de la educación. Madrid: Alianza.

Novak, J. (1998). Learning, Creating and Using Knowledge. New Jersey: Lawrence Erlbaum Associates.

Novak, J. y Gowin, (1999). Aprendiendo a aprender. Barcelona: Ed. Martínez Roca.

Orozco Rodríguez, C., Morales Morgado, E. M., Gonçalves da, F. (2014). The eXeLearning and GeoGebra integration for teaching geometrics definitions and vectors representations through Learning Objects. En ActasSecond International Conference on Technological Ecosystems for Enhancing Multiculturality TEEM'14. Recuperado de http://dx.doi.org/10.1145/2669711.2669967.

Orozco Rodríguez, C., Morales Morgado, E. y Campos Ortuño, R. (2016). Creación de Objetos de Aprendizaje basados en la teoría de los Modelos Mentales de Johnson-Laird. Série-Estudos, (21), 42, p. 2139. http://dx.doi.org/10.20435/2318-1982-2016-v.21-n.42(02).

Pea, R. (2001). Prácticas de inteligencia distribuida y diseños para la educación. En G. Salomon (Comp.), Cogniciones distribuidas. Consideraciones psicológicas y educativas. Buenos Aires: Amorrortu.

Postman, N. \& Weingartner, Ch. (1969). Teaching as a subversive activity. New York: Dell Publishing Co.

Pozo, J. I. (1989). Teorías cognitivas del aprendizaje. Madrid: Morata.

Rahmani, R. (2016). Toward developing and validating a model for implementation of learning objects. The Online Journal of New Horizons in Education, Vol. 6 (4), 270-289. 